\title{
Percepción alofónica en la dislexia: una revisión
}

\section{Allophonic Perception in Dyslexia: An overview}

\author{
Willy Serniclaes \\ Laboratoire Psychologie de la Perception, CNRS \& Université Paris Descartes, France.
}

Disponible online 31 de agosto de 2011

\begin{abstract}
La teoría alofónica defiende que las personas afectadas por dislexia perciben el habla mediante unidades alofónicas en lugar de unidades fonémicas. Después de revisar la teoría de rasgos de la percepción del habla se presenta un resumen de la evidencia que apoya la teoría alofónica. Se enfatiza la diferencia entre la percepción alofónica y la reducción de la agudeza en la percepción de fonemas, siendo esta última una característica común en muchos problemas de desarrollo del lenguaje y, por tanto, no específica de la dislexia. Teniendo en cuenta esta diferencia fundamental, se examina la evidencia contraria a la teoría alofónica y se formulan propuestas para futuras evaluaciones e intervenciones.

Palabras clave: Dislexia; Teoría Alofónica; Percepción Categorial; Agudeza Fonémica.

Allophonic theory claims that people affected by dyslexia perceive speech with allophonic rather than phonemic units. After a reminder about the featural theory of speech perception, the evidence supporting the allophonic theory is summarized. The difference between allophonic perception and reduced phoneme perception acuity is emphasized, the latter being a common characteristic of various troubles of language development and is thus not specific to dyslexia. Taking account of this fundamental difference, the counter-evidence against allophonic theory is examined and proposals for future testing and remediation are formulated.
\end{abstract}

Keywords: Dyslexia; Allophonic Theory; Categorical Perception; Phonemic Acuity.

Correspondencia: Willy Serniclaes. Laboratoire Psychologie de la Perception, CNRS y Université Paris Descartes, France. E-mail: willy.serniclaes@ parisdescartes.fr 
En una investigación sobre la percepción categorial de los niños disléxicos encontramos que éstos perciben diferencias entre variantes acústicas del mismo fonema (por ej.: dos $/ \mathrm{b} /$ diferentes) con una mayor agudeza que los niños neurotípicos (Serniclaes, Sprenger-Charolles, Carré y Démonet, 2001). Además, encontramos que los niños disléxicos eran especialmente sensibles a las variantes alofónicas del mismo fonema, por ej.: a dos sonidos diferentes que pertenecen a diferentes categorías fonémicas en otras lenguas (Serniclaes, Van Heghe, Mousty, Carré y Sprenger-Charolles, 2004). Estos hallazgos eran bastante inusuales en vista del amplio repertorio de déficits asociados a la dislexia. La conciencia fonémica, la memoria fonológica, atención, audición, visión, ... en todas ellas se ha mostrado, con mayor o menor éxito, que la gente afectada con problemas específicos de lectura es deficiente (para una revisión véase: Sprenger-Charolles, Colé y Serniclaes, 2006). Contrariamente, nosotros encontramos que los disléxicos se comportaban mejor que los neurotípicos en la percepción de alófonos y que su rendimiento mejorado, a pesar de sus potenciales ventajas para aprender una lengua extranjera, representaba un hándicap específico para aprender a leer. Percibir los alófonos de un mismo fonema como unidades distintas perturba el principio alfabético mediante el cual cada grafema está idealmente asociado con un fonema (como en español) y, por tanto, tiene implicaciones directas para la adquisición de la lectura.

Aquí, haré una revisión de la evidencia a favor y en contra de la teoría alofónica de la dislexia. La acumulación de diferentes hallazgos servirá para formular una nueva síntesis teórica y nuevas perspectivas para la intervención. Después de repasar la teoría de rasgos de la percepción del habla, haré un resumen de la evidencia en apoyo de la teoría alofónica. Entonces, explicaré la diferencia entre la percepción alofónica y la reducción de la agudeza en la percepción de fonemas, siendo esta última una característica común a diferentes problemas de lenguaje, por tanto, no específica de la dislexia. Teniendo en cuenta esta diferencia fundamental, examinaré la evidencia en contra de la teoría alofónica y formularé propuestas para futuras evaluaciones e intervenciones.

\section{Rasgos y fonemas en la percepción del habla} Desarrollo temprano de la percepción del habla en la infancia: acoplamiento entre los umbrales psicoacústicos universales

Los constituyentes básicos de los sistemas fonológicos son unidades diferenciales, no fonemas, sino oposiciones entre fonemas (Jakobson, 1973, p.130). Un fonema puede tomar múltiples formas acústicas diferentes, dependiendo de los fonemas circundantes, pero la diferencia entre dos fonemas permanece constante. Por ejemplo, lo que distingue /p/ de /z/ es un conjunto de propiedades o "rasgos": un inicio tardío de la voz (rasgo de "sonoridad"), bajas frecuencias de los formantes ("punto de articulación") y duración corta ("modo de articulación").
Los valores absolutos del tiempo de inicio de la sonoridad vocálica $\left(\mathrm{TIV}^{1}\right)$, los valores absolutos de las frecuencias de los formantes y la duración tanto de /p/ como de / $\mathrm{z} /$ varían con el contexto vocálico, pero las diferencias relativas al TIV, la frecuencia y la duración, permanecen considerablemente constantes. De acuerdo con la teoría de los rasgos distintivos, estas diferencias son invariantes en algún nivel del procesamiento perceptivo.

Suponiendo que los rasgos son de hecho invariantes perceptivas y dado que los fonemas pueden ser definidos como "haces" de rasgos (Clements, 1985), éstos últimos son potencialmente invariantes. Sin embargo, los rasgos son propiedades universales, independientes del lenguaje, mientras que los fonemas son específicos de cada lengua, y agrupar los rasgos universales en los fonemas específicos del lenguaje es un proceso bastante complejo.

Para ilustrar este aspecto, tomemos el ejemplo del rasgo de sonoridad. Diferentes pruebas indican que la percepción del TIV está anclada en diferentes umbrales o "límites" psicoacústicos (para adoptar la terminología común en esta área de investigación). La sensibilidad a los límites universales de TIV, localizados entre TIVs de $-30 \mathrm{y}+30 \mathrm{~ms}$ ha sido demostrada en estudios conductuales con análogos de TIV que no pertenecen al habla (Pisoni, 1977), estudios electrofisiológicos con monos (Sinnott y Gillmore, 2004), potenciales evocados ante estímulos de habla con participantes humanos (Hoonhorst, Colin, Deltenre, Radeau y Serniclaes, 2009a) y experimentos con niños pre-lingüísticos (con menos de 6 meses e independientemente del contexto lingüístico: en un contexto español: Lasky, Syrdal-Lasky y Klein, 1975; en un contexto inglés: Aslin, Pisoni, Hennessy y Perry, 1981; en un contexto francés: Hoonhorst, Colin, Deltenre, Radeau y Serniclaes, 2009b). Ahora, estos +-30 ms de límites de TIV se usan para separar fonemas en lenguas con tres categorías de sonoridad (por ej.: Thai: Lisker y Abramson, 1970). Sin embargo, en otras muchas lenguas con sólo dos categorías de sonoridad (español, francés, holandés, ...) el límite fonémico se localiza en el TIV de $0 \mathrm{~m}$ y este límite aparece más tarde durante el desarrollo perceptivo, después de los 6 meses de edad (en español: Eilers, Gavin y Wilson, 1979; en francés: Hoonhorst et al., 2009b). Este ejemplo de percepción de la sonoridad ilustra cómo un límite fonémico deriva de los límites universales. Es necesario señalar que el límite fonémico, que corresponde con la percepción del orden temporal entre dos eventos $(0 \mathrm{~ms}$ es el límite entre la anticipación y el retardo) es intrínsecamente más complejo que los límites universales, que corresponden con la percepción de una anticipación o un retardo (Figura 1). La adquisición de este límite fonémico es el resultado de "acoplar" el umbral psicoacústico, por ej.: a partir de dependencias cruzadas en la percepción de estos umbrales (Serniclaes, 2011).

1. En ingles voice onset time ó VOT. 
Figura 1. (Adaptada de Serniclaes et al., 2004). Límites de TIV universales $(\mathrm{a}-30 \mathrm{y}+30 \mathrm{~ms})$ corresponden a la percepción de una anticipación o un retardo. El límite de TIV 0 es específico de algunas lenguas (incluidas el español, el francés, el holandés...).

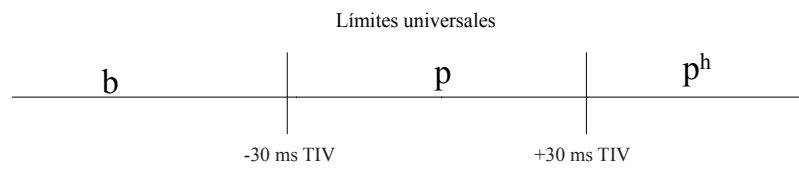

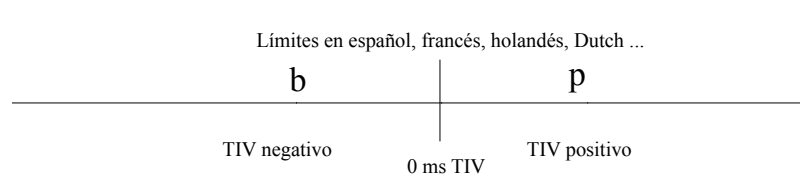

Los acoplamientos entre los límites psicoacústicos universales no son específicos de la sonoridad. La percepción del contraste del punto de articulación entre las consonantes sordas depende de la transición del segundo y el tercer formante (transiciones F2 y F3). Los límites del punto de articulación en los espacios de transición de las F2 y F3 están relacionados con los cambios ascendentes/descendentes en la dirección de las transiciones F2 y F3, que constituyen límites psicoacústicos universales (para una revisión véase Serniclaes, 2011). Estos límites universales se combinan de modo específicos para percibir el punto de articulación en diferentes lenguas (Serniclaes y Geng, 2009).

Aunque puede ser complejo, los acoplamientos fonémicos entre los límites universales parecen emerger espontáneamente después de que el niño ha sido expuesto durante varios meses a los sonidos del lenguaje del entorno.

Sin embargo, algunos niños pueden no desarrollar estos acoplamientos fonémicos por razones genéticas. Estos niños adquirirían un modo "alofónico" de percepción del habla, esto es, percibirían categorías universales que se usan a veces como fonemas en otras lenguas (por ej.: las tres categorías de voz del Thai). Al carecer de representaciones fonémicas, los niños que desarrollan una percepción alofónica presentarían más adelante problemas específicos de lectura.

Desarrollo posterior de la percepción del habla durante la niñez y la adolescencia: adquisición de claves acústicas secundarias

La percepción de rasgos fonémicos de los adultos no se basa sólo en los acoplamientos entre los límites universales. Estos límites desempeñan un papel fundamental en la percepción de un rasgo fonémico dado, pero también contribuyen a la percepción del mismo rasgo fonémico diferentes claves acústicas, aunque tengan un peso perceptivo menor. Por ejemplo, en francés el límite TIV promedio se localiza a los $0 \mathrm{~ms}$, pero un incremento/ decremento en el tono (F0) cambia este límite hacia TIVs positivos/negativos (Serniclaes, 1987). Estos cambios en la percepción del TIV son paralelos a los observados en la producción de
TIV: el TIV es prolongado, haciendo el percepto más sordo; en contextos en los que la F0 es más baja, el percepto se hace más sonoro. La integración de claves secundarias como la F0 en la percepción de la sonoridad compensa la variación contextual de TIV (debido a la coarticulación). Estas “compensaciones de la coarticulación" (para una revisión reciente véase Mitterer, 2006) contribuyen a la invarianza perceptiva del rasgo.

La integración de claves acústicas secundarias en la percepción de rasgos ocurre tardíamente en el curso del desarrollo. La emergencia de los acoplamientos fonémicos, antes del primer año, es seguida de una evolución lenta que sólo termina durante la adolescencia. Los acoplamientos entre los límites universales no generan representaciones de fonema completas, comparables a las encontradas en los adultos. Esto fue lo que se observó en estudios de la percepción de sonidos del habla que variaban entre dos fonemas diferentes a lo largo de un continuo acústico (por ej.: un continuo de TIV entre $/ \mathrm{b} / \mathrm{y} / \mathrm{p} /$ ). Comparado con los adultos, las funciones de identificación de los niños muestran efectos suelo/techo al final de continuo, es decir, valores asintóticos por debajo de la identificación perfecta (véase Figura 2 de Medina, Hoonhorst, Bogliotti y Serniclaes, 2010; para una revisión véase, Hoonhorst, Medina, Colin, Markessis, Radeau, Deltenre y Serniclaes, 2011). Tales diferencias en los valores asintóticos de las funciones de identificación surgen de una ponderación perceptiva inapropiada, tal como demostró Treisman (1999). Con ponderaciones apropiadamente bajas estas claves sólo afectan a la localización del límite fonémico, situado en algún lugar en el medio del continuo, pero cuando se sobrevaloran pueden generar asíntotas por debajo/encima de $100 \% / 0 \%$ en los límites finales del continuo. De este modo, la sobrevaloración de las claves acústicas secundarias tiene el efecto de reducir la precisión de la identificación del fonema.

Figura 2. (adaptada de Medina et a., 2010). Propiedades categoriales. Estas propiedades se ilustran con las curvas de identificación y discriminación hipotéticas. La precisión del límite es mayor cuando las curvas de identificación son más empinadas (compárese Fig. 2a con Fig. 2d) o, de modo equivalente, cuando los picos de discriminación son más elevados (Fig. 2b con Fig. 2e; Fig 2c con Fig. 2f). La percepción categorial es mayor cuando los picos observados y esperados coinciden (compárese Fig. 2b con Fig. 2c; Fig 2e con Fig. 2f).

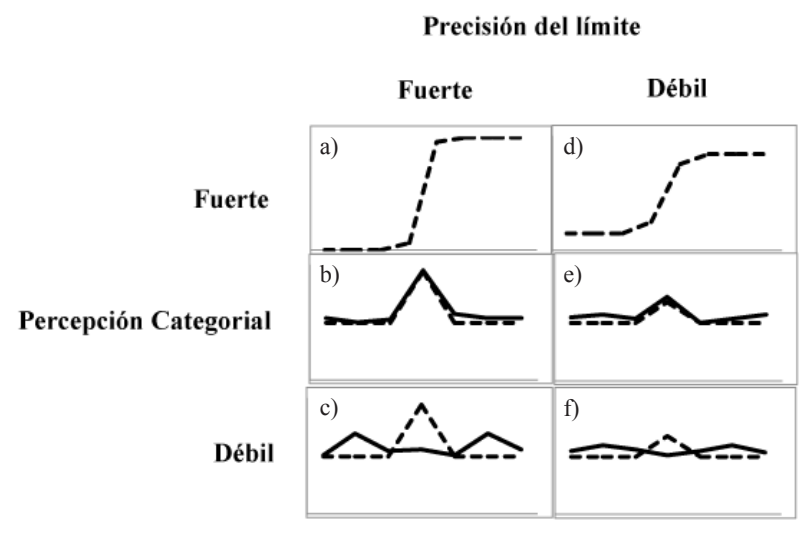


Los niños con patologías de lenguaje muestran un grado menor de precisión en la percepción de rasgos fonémicos cuando se comparan con niños neurotípicos de la misma edad. Esto no sólo es válido para los disléxicos, sino también, por ejemplo, para niños sordos con implantes cocleares (Bouton, Serniclaes, Coléy Bertoncini, aceptado; Medina y Serniclaes, 2009). Contrariamente a lo que ocurre con la percepción alofónica, el déficit en la precisión perceptiva no es específico de la dislexia. Es una cuestión de retraso evolutivo más que de desviación.

\section{Percepción alofónica \\ Pruebas a favor de la percepción alofónica}

Los niños disléxicos son mejores en la percepción intracategorial que los lectores normales (Serniclaes et al., 2001) y son más sensibles a los contrastes alofónicos pertenecientes a una categoría fonémica (Serniclaes et al., 2004), lo que sugiere un modo específico de percepción basado en alófonos más que en fonemas. Este modo de percepción del habla puede constituir un serio obstáculo para la formación de asociaciones grafemafonema, necesarias para adquirir una lectura fluida.

El incentivo para postular un modo alofónico de percepción de habla en niños proviene de la investigación sobre la percepción categorial. La percepción categorial (CP) significa que sólo se pueden percibir diferencias entre categorías, no variaciones dentro de la categoría (Liberman, Harris, Hoffman y Griffith, 1957). Nótese que la percepción del habla deja de ser perfectamente categorial con frecuencia y que el grado de PC depende de varios factores (Harnad, 1988). De modo notable, los niños afectados por dislexia han mostrado sufrir un déficit en PC de los sonidos del habla en un numero cuantioso de estudios, empezando por el de Brandt y Rosen (1980). Sin embargo, el interés teórico del déficit de PC sólo se ha hecho patente recientemente cuando se ha mostrado que los disléxicos no sólo tienen una discriminación más pobre entre categorías, sino una mejor discriminación dentro de las categorías (Serniclaes et al., 2001). En lugar de ser un problema de resolución débil, como son los déficit asociados con la dislexia, que asumen capacidades sensoriales reducidas, los déficit de PC surgen de una sobrediscriminación de diferencias entre estímulos que no son funcionales para fines lingüísticos. Tales diferencias son de naturaleza "alofónica" en el sentido de que corresponden a distinciones que son meras variantes contextuales de fonemas en una lengua dada, aunque sean fonémicas en otras lenguas (Bogliotti, Serniclaes, Messaoud-Galusi, y Sprenger-Charolles 2008; Burnham, 2003; Serniclaes et al., 2004; Luque, Serniclaes, López-Zamora, Bordoy, Giménez, Rosales y Varona, 2011; véase Figura 3).

Figura 3. Identificación (izquierda) y discriminación (derecha) de contrastes de TIV por niños españoles disléxicos y sus controles (7 y 9 años) en un continuo ba/pa. Los valores asintóticos de la función de identificación exhiben efectos acusados de suelo/techo en los disléxicos vs los controles, lo que refleja una precisión perceptiva más débil. Los controles muestran un pico mayor de discriminación en el límite fonológico (TIV $0 \mathrm{~ms}$ ). Los disléxicos muestran tres picos diferentes, uno en el límite fonológico, los otros dos en los límites universales $(-30$ y +30 $\mathrm{ms})$ lo que indica percepción alofónica.
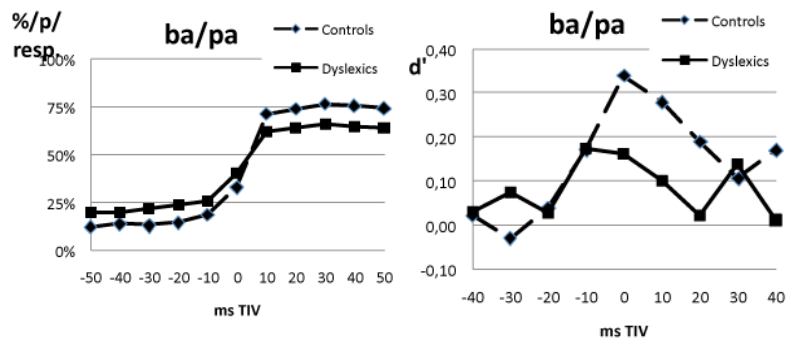

El déficit de PC revelaría de este modo una forma "alofónica" de percepción del habla caracterizada por el uso de alófonos más que de fonemas. La inflación del repertorio fonológico en los disléxicos tiene implicaciones evidentes para la adquisición de la lectura porque, incluso en lenguas con un sistema de lectura transparente (por ej.: el español), percibir el habla con alófonos en lugar de fonemas hace confusa la correspondencia uno a uno entre letras y fonemas.

\section{Origen evolutivo de la percepción alofónica}

Ya que la percepción de distinciones alofónicas no es necesaria para reconocer las palabras habladas uno puede preguntarse sobre su origen en el curso del desarrollo del lenguaje. Tal como hemos visto, el niño no tiene que aprender estas distinciones porque corresponden a límites universales que están presentes desde el nacimiento. Sólo después de ser expuestos a los sonidos de su lengua nativa, los niños adquieren contrastes fonológicos específicos de la lengua mediante el acoplamiento entre límites universales. Recuérdese que para el rasgo de sonoridad, el TIV se sitúa en los 0 ms en lenguas como el francés, el español y el polaco (Ganong y Keating, 1981; Medina et al., 2010; Williams, 1977), un valor que no está incluido en los límites universales (localizados en el TIV de +/-30), aunque no aparece demasiado pronto en el curso del desarrollo del lenguaje (Eilers et al., 1979; Hoonhorst et al., 2009b). Esto significa que es preciso adquirir un nuevo límite, irreductible a uno de los dos límites naturales y que caiga justo entre estos dos límites. El proceso que hace posible tal adquisición es bastante complejo y requiere el acoplamiento entre dos mecanismos universales.

Ya que el acoplamiento entre predisposiciones es un proceso bastante complejo, no es sorprendente que pueda fallar en una parte de la población, dando lugar a representaciones de los sonidos de habla alofónicas en lugar de fonológicas. Esta idea viene apoyada por estudios genéticos que sugieren una base hereditaria para la dislexia (para una revisión: Démonet, Taylor y Chaix, 2004).

La percepción alofónica probablemente no dificulta en ningún modo significativo el reconocimiento de la palabra hablada, aunque puede ser almacenada de un modo más costoso debido al procesamiento de información redundante. Las consecuencias de la percepción alofónica son mucho más importantes para la adquisición de la lectura. Un niño que utilice categorías alofónicas en lugar de fonémicas encontrará considerablemente más dificultades para establecer correspondencias entre fo- 
nemas y grafemas, incluso en una lengua con una ortografía completamente transparente. De hecho, incluso aunque tal lengua pueda ofrecer correspondencias uno a uno entre fonemas y grafemas, un niño que perciba en alófonos tendrá que enfrentarse con correspondencias muchos a uno, ya que existen varios alófonos para cada fonema. Las simulaciones computacionales apoyan la hipótesis de una relación causal entre el déficit de PC y la dislexia mostrando que la supresión de "atractores fonológicos" entre rasgos fonéticos, conceptualmente similar a los "acoplamientos fonológicos" definidos anteriormente, tiene unos importantes efectos negativos en el rendimiento lector de una red conexionista (Harm y Seidenberg, 1999). Esto apoya el argumento de que la percepción alofónica puede afectar de modo severo al rendimiento lector de los seres humanos. Finalmente, un estudio con analfabetos adultos mostró que no tienen un déficit de PC (Serniclaes, Ventura, Morais y Kolinsky, 2005), indicando que la percepción alofónica es la causa, más que la consecuencia, de la dislexia.

\section{Percepción alofónica vs agudeza perceptiva reducida}

La percepción alofónica es una discrepancia entre el proceso de identificación basado en límites fonológicos específicos y los procesos de discriminación basados en límites psicoacústicos universales. Los disléxicos no sólo muestran una percepción alofónica, también tienen una menor agudeza tanto para la discriminación como para la identificación de rasgos fonológicos. La diferencia entre una agudeza perceptiva reducida y una percepción alofónica es que la primera consiste en un déficit cuantitativo, un problema de valoración de claves secundarias irrelevantes para un rasgo fonológico dado, tal como ha sugerido Treisman (1999), mientras que la percepción alofónica implica un déficit cualitativo en la integración de las claves acústicas que desempeñan un papel importante en la percepción de rasgos.

Un déficit en la agudeza perceptiva se refleja en un pico de discriminación reducido, una curva más suave de la función de identificación y también por las puntuaciones de identificación asintóticas en el suelo y en el techo (Figura 2). Muchos estudios han mostrado que los disléxicos producen curvas de función de identificación menos pronunciadas, o equivalentes a un punto de discriminación menor, en algunos continuos estimulares, aunque otros estudios no han encontrado diferencias significativas (para una revisión véase: Vandermosten, Boets, Luts, Poelmans, Wouters y Ghesquière, 2011).

Sin embargo, la agudeza de la percepción del rasgo no sólo depende del estatus de lectura, sino también de otros factores como la edad o el estatus auditivo. Diversos estudios han mostrado que la precisión categorial cambia en función de la edad en los niños normales (para una revisión véase: Hoonhorst et al., 2011). También, los niños sordos con implante coclear muestran una menor precisión categorial que los niños con audición normal con la misma experiencia auditiva (Bouton et al., aceptado; Medina y Serniclaes, 2009). Por el contrario, no hay efecto ni de la edad ni del estatus auditivo en la percepción categorial (sin efecto de la edad: Hoonhorst et al., 2011; Medina et al., 2010; sin efecto del estatus auditivo: Bouton et al., aceptado; Medina y Serniclaes, 2009). Nótese, sin embargo, que se ha puesto de manifiesto un déficit de precisión en adultos disléxicos (Ruff, Marie, Celsis, Cardebat y Démonet, 2003; van Beinum, Schwippert, Been, van Leeuwen, y Kuijpers, 2005; Vandermosten, Boets, Luts, Poelmans, Golestani, Wouters y Ghesquière, 2010), lo que sugiere que no es sólo una cuestión de retraso, sino de desviación.

Para resumir, existen abundantes pruebas de que los disléxicos manifiestan un déficit en la precisión perceptiva. Sin embargo, este déficit no es específico de la dislexia y es en parte un problema de retraso en el desarrollo. No debe confundirse con la percepción alofónica que es específica de la dislexia, o con niños con múltiples déficit que incluyen problemas de lectura (niños disfásicos: Zobouyan, Bertoncini y Serniclaes, 2010; niños con el síndrome de William: Majerus, Poncelet, Bérault, Audrey, Zesiger, Serniclaes, y Barisnikov, 2011).

\section{Sustrato neurológico de la percepción alofónica}

Aunque la percepción alofónica en niños disléxicos ha sido probada en al menos cinco estudios conductuales (Bogliotti et al., 2008, Burnham, 2003; Luque et al., 2011; Noordenbos, Segers, Mitterer, Serniclaes y Verhoeven, 2010; Serniclaes et al., 2004), también hay hallazgos negativos. En un estudio sobre la percepción entre TIV cortos y largos, que es fonémico en coreano, pero alofónico en francés, Ramus y Szenkovits (2008), mostraron de que los niños disléxicos franceses no hacían una sobrediscriminación para este contraste.

Sin embargo, la ausencia de percepción alofónica en las respuestas conductuales no evita una carencia del sustrato neuronal. En un estudio de seguimiento con niños holandeses con un continuo a bə/də, Noordenbos et al. (2010) no hallaron percepción alofónica en niños con riesgo familiar de dislexia, mientras que los mismos niños habían mostrado percepción alofónica en la guardería. Sin embargo, un estudio posterior con potenciales evocados puso en evidencia un incremento de Mismatch Negativity (MMN) en el límite alofónico (Noordenbos et al., enviado). Esto sugiere que la gente con riesgo familiar de dislexia, incluyendo aquellos que desarrollaran dislexia más tarde, procesan los contrastes de habla mediante vías neuronales alofónicas, incluso cuando utilizan estrategias alternativas para responder a las demandas de las tareas conductuales.

Los correlatos neuronales, tanto la percepción fonémica como la alofónica, se pusieron de manifiesto en tres estudios relacionados en los que se utilizó el mismo material estimular. Se tomaron las respuestas neuronales a ondas análogas de las sílabas ba/da con fMRI en adultos neurotípicos (Dehaene-Lambertz, Pallier, Serniclaes, Sprenger-Charolles, Jobert y Dehaene, 2005) y mediante PET tanto en adultos neurotípicos como disléxicos (Dufor, Serniclaes, Sprenger-Charolles, y Démonet, 2007; 2009). El interés de las ondas análogas es que los oyentes inexpertos las perciben espontáneamente como silbidos no lingüísticos, pero estas mismas ondas son percibidas como 
sonidos de habla después de una sesión informativa (Remez, Rubin, Pisoni y Carrell, 1981). Esto permite comparar las respuestas conductuales y neuronales en dos modalidades diferentes, habla versus no habla, con exactamente el mismo material, evitando así distractores acústicos. Los resultados de los dos estudios de onda se presentan en la figura 4. El estudio de fMRI (Figura 4a) mostró que el cambio de modalidad de no habla a habla generó un incremento en la actividad neuronal ante los diferentes contrastes estimulares sólo en una región: el gyrus supramarginal izquierdo (GSM), una región parietal localizada en la vía motora-fonológica ("dorsal") de la percepción del habla. El primer estudio de PET de Dufor et al (2007) también reveló un incremento específico del habla en la discriminación fonémica en el GSM izquierdo en adultos neurotípicos, pero no en disléxicos (Figura 4b). El hecho de que no se hallaran diferencias en la modalidad de no-habla sugiere que los disléxicos no tienen déficit en la percepción de límites psicoacústicos, contrariamente a lo que defienden las teorías auditivas (más recientemente: Vandermosten et al., 2010; 2011).

En un análisis posterior de los datos de PET del segundo estudio, Dufor et al. (2009) demostraron diferencias sutiles entre adultos neurotípicos y disléxicos en las respuestas a los contrastes intrafonémicos. Estas diferencias se localizaban en la región prefrontal (opérculo frontal izquierdo) cercana al área de Broca (Figura 5). Los disléxicos adultos eran más "alofónicos" (discriminaban mejor pares intracategoriales) cuando activaban el opérculo frontal izquierdo, mientras que los controles eran menos "alofónicos" aun cuando activaban las mismas áreas. Parecería que la percepción alofónica sólo prevalece cuando se percibe el habla en relación a representaciones motoras (a lo largo de la "corriente dorsal" de Hickok y Poeppel, 2007) lo que recuerda que leer en alto es "el sine qua non de la adquisición de la lectura" (Share, 1995).
Figura 5. Diferencias en la actividad neuronal entre la modalidad no habla y habla entre adultos disléxicos y neurotípicos, principalmente en la región premotora izquierda (1* en el gráfico adaptado de Dufor et al., 2009).
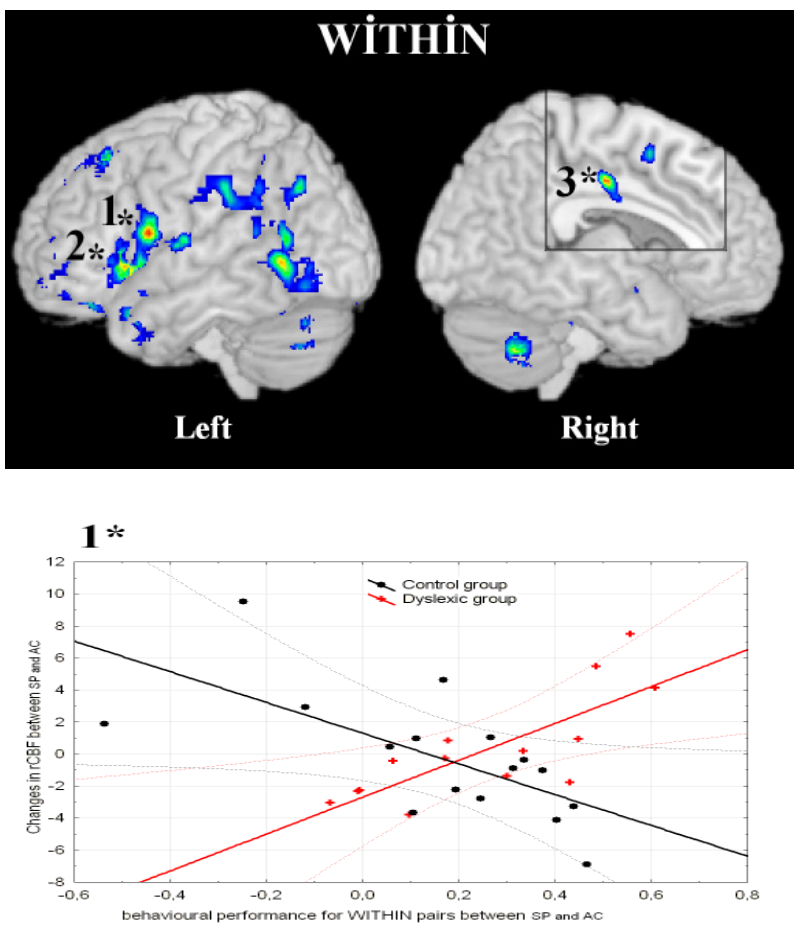

Marco alofónico

La acumulación de pruebas empíricas sobre la percepción alofónica y, más generalmente, el progreso de los procesos cerebrales implicados en la lectura, sugiere una modificación importante del modelo alofónico. El modelo de procesamiento de la información que propusimos en 2004 (Serniclaes et al., Fig.1)

Figura 4. Diferencias en la actividad neuronal entre la modalidad de habla y de no habla en el giro supramarginal: en los adultos neurotípicos en un estudio de FMRI (Figura 4 a, adaptado de Dufor et al., 2007); en adultos disléxicos vs neurotípicos en un estudio de PET (Figura 4b, adaptada de Dehaene-Lambertz et al., 2005).

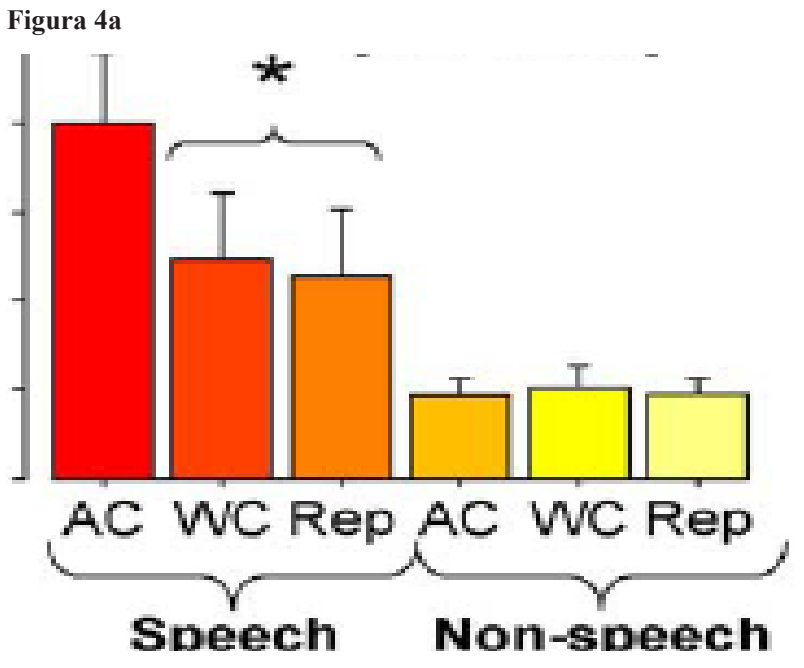

Figura 4b

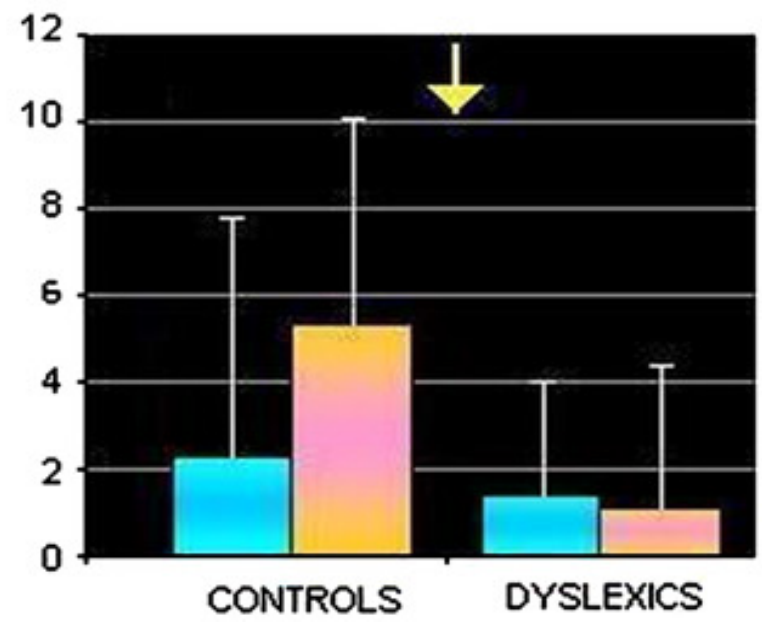


ha sido modificado para incorporar tres cambios principales. Una primera modificación viene motivada por el hecho de que el nivel de procesamiento fonético, entre los niveles acústico y fonológico, no parece ser necesario. Tal como se explica más arriba, los rasgos alofónicos universales corresponden a umbrales psicoacústicos naturales en lugar de ser específicos del habla (Serniclaes, 2011). Por tanto, el estadio de procesamiento fonético no se incluye en el modelo que se presenta en la Figura 6.

Figura 6. Estructura alofónica en el cerebro. Vía dorsal: en el procesamiento neurotípico, los sonidos de habla son categorizados en categorías alofónicas en el cortex auditivo y se combinan en categorías fonémicas en el cortex prefrontal izquierdo (Dufor et al., 2009) después de pasar a través del giro supramarginal (Dufor et al., 2007). Durante la adquisición de la lectura los fonemas se asocian a letras en un área de integración auditivo-visual (en un área que incluye el planum temporal: Blau, van Atteveldt, Ekkebus, Goebel y Blomert, 2009; Blau, Reithler, van Atteveldt, Seitz, Gerretsen, Goebel, y Blomert, 2010). En las personas disléxicas, falta la conversión de alófono a fonema y los alófonos se envían al área de integración. Vía ventral: la integración letra-fonema ocurre en el interfaz léxico (en un área que incluye el giro temporal medio Hickok y Poeppel, 2007). En el lexicón, la representación de fonemas es menos categorial, más dependiente del contexto, lo que sugiere que los fonemas se agrupan en cadenas (McMurray, Tanenhaus y Aslin, 2002).

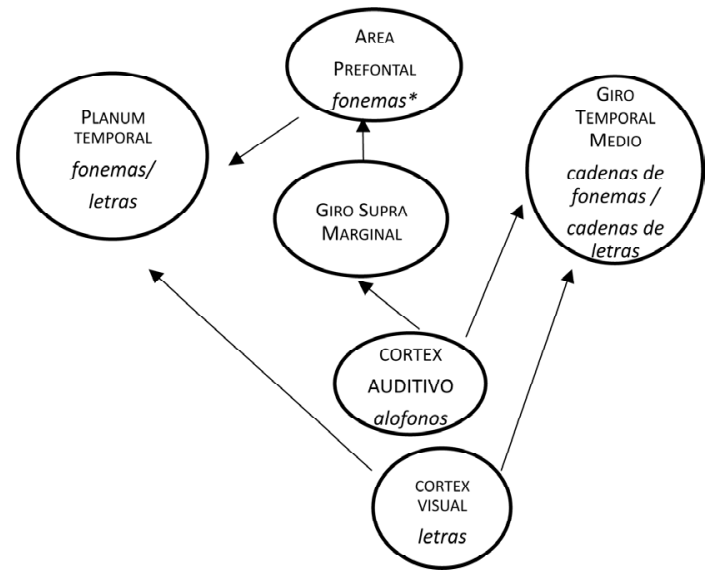

Una segunda modificación está originada por el hecho de que la diferencia crucial entre las representaciones fonológicas de lectores disléxicos y neurotípicos, fonémicas versus alofónicas, se localiza en el área premotora izquierda (Dufor et al., 2009). Consecuentemente, el lugar de la diferencia en el procesamiento fonológico es ahora más específico y está localizado en esta área. Una tercera modificación está motivada por el descubrimiento de un área integradora del sonido-letra en la vecindad del cortex auditivo (giro temporal antero-superior, Planum temporal/Sulcus Heschl y Sulcus temporal superior: Blau et al., 2010). Consecuentemente, las implicaciones de la percepción alofónica de la lectura deberían localizarse en esta área integradora de sonido-letra. Sin embargo, la mera integración de sonidos de habla y letras generaría problemas para la lectura porque los sonidos de habla se categorizan en categorías alofónicas más que fonémicas sin transición a través del área premotora izquierda. Por tanto, se ha añadido al modelo un flu- jo arriba-abajo del área premotora izquierda al área integradora del sonido-letra. Este flujo arriba-abajo llevaría fonemas en los neurotípicos, pero llevaría alófonos sin transformar en los disléxicos, explicando de este modo su problema específico de lectura. Finalmente, con los sistemas de ortografía completamente transparentes, las letras pueden ser utilizadas como unidades visuales en el área integradora. Sin embargo, en los otros sistemas, las letras tienen que ser recodificadas en grafemas con el fin de obtener la relación uno a uno con los fonemas.

\section{Perspectivas para la intervención}

Independientemente de lo convincente de los argumentos previos a favor de una teoría de la dislexia, el mayor desafío es si pueden o no remediar el déficit de lectura de los disléxicos. Ha habido, al menos, seis intentos de remediar la dislexia mediante el aprendizaje de la percepción fonémica. Hurford (1990) defendió que el entrenamiento en la discriminación de pares mínimos de fonemas mejoraba la capacidad metafonológica evaluada mediante una tarea de sustracción de fonemas. Veuillet, Magnan, Ecalle, Thai-Van y Collet (2007) también utilizaron el entrenamiento en la discriminación de contrastes mínimos de fonemas y encontraron tanto mejoras conductuales (localización del límite de TIV) como neuronales (actividad asimétrica en la oliva coclear media) en niños disléxicos después del entrenamiento con implicaciones para la lectura. Van Heghe (2001) entrenó a niños disléxicos para que discriminasen diferencias entre fonemas e ignorasen diferencias acústicas dentro de las categorías fonémicas. Sorprendentemente, el entrenamiento no mejoró la percepción categorial, pero mejoró sus capacidades metafonológicas. Sin embargo, no se pudo excluir un efecto test-retest porque el tamaño del grupo control era demasiado pequeño para permitir confirmar las conclusiones. Bogliotti (2005) también entrenó a niños disléxicos para que discriminaran diferencias entre fonemas, pero, en lugar de enseñar a los niños a ignorar diferencias intracategoriales, ella les enseñó a identificarlas con el mismo rótulo (siguiendo un procedimiento iniciado por Guenther, Husain, Cohen y Shinn-Cunningham, 1999; Guenther y Bohland, 2002). El entrenamiento mejoró la precisión de la identificación de fonemas, pero no modificó la percepción categorial en torno al límite fonémico. Sin embargo, y de nuevo sorprendentemente, el entrenamiento produjo picos de discriminación alrededor de los límites alofónicos (Bogliotti y Serniclaes, 2011). Collet, Serrniclaes, Colin y Leybaert (2011) entrenaron a niños disfásicos con una tarea de discriminación basada en el paradigma de fading perceptivo, que consiste en reducir progresivamente el tamaño de la diferencia acústica entre dos estímulos situados en el límite fonémico (Jamieson y Morosan, 1986). Los resultados mostraron la emergencia de picos de discriminación en los límites de TIV alofónicos $(-30 \mathrm{y}+30 \mathrm{~ms})$, seguida de una mejora en la percepción categorial alrededor del límite de TIV fonémico ( 0 ms tanto en francés como en español). Es más, el entrenamiento mejoró la conciencia fonémica, lo que sugiere posibles implicaciones para el rendimiento en la lectura. Finalmente, 
Chobert (2011) mostró que: (1) los niños disléxicos muestran picos de MMN similares para las diferencias de TIV inter e intracategoriales, contrariamente a los niños neurotípicos que muestran un mayor MMN para las diferencias de TIV intercategorial que para el intracategorial; (2) el entrenamiento musical mejoró el MMN fonémico en los niños disléxicos, aunque esta mejora no se generalizó a la conciencia fonémica.

En conjunto estos diferentes intentos de mejorar el rendimiento específico de la lectura apuntan a una posible solución con un método bien diseñado para mejorar la percepción categorial. Al menos tres factores tienen un interés en este propósito: la elección de contrastes de sonido, que tiene que ser suficientemente complejo para activar acoplamientos fonémicos, el entrenamiento discriminativo, que debería proceder con cada vez mayores niveles de complejidad, y lo que debería denominarse un "acercamiento lateral" para mejorar la percepción del habla mediante la transferencia de actividades de otros dominios cognitivos.

\section{Conclusiones}

En pocas palabras: (1) existe abundante evidencia tanto conductual como neuronal para mostrar que los niños disléxicos tienen una mejor percepción de los contrastes de habla alofónica que los niños neurotípicos; (2) la localización neurológica de la percepción alofónica está próxima al área de Broca; (3) la percepción alofónica puede aparecer en los datos neuronales, aun en ausencia de datos conductuales; (4) la mejora de la percepción alofónica es posible y existen algunas pruebas que sugieren que puede remediar la dislexia.

\section{References}

1. Abramson, A.S. \& Lisker, L. (1970). Discriminability along the voice onset time continuum: cross-language tests. In B.Hala, M. Romportl \& P. Janota Eds., Proceedings of the 6th International Congress of Phonetic Sciences, Prague 1967. Prague: Academia; 569-573.

2. Aslin, R.N. (1989). Discrimination of frequency transitions by human infants. Journal of the Acoustical Society of America, 86, 582-590. http://dx.doi.org/10.1121/1.398237

3. Aslin, R.N., Pisoni, D.B., Hennessy, B.L. \& Perey, A.V. (1981). Discrimination of voice onset time by human infants: New findings and implications for the effect of early experience. Child Development, 52, 1135-1145. http:// dx.doi.org/10.2307/1129499

4. Blau, V., van Atteveldt, N., Ekkebus, M., Goebel, R. \& Blomert, L. (2009). Reduced neural integration of letters and speech sounds links phonological and reading deficits in adult dyslexia. Current Biology, 19, 503-508. http:// dx.doi.org/10.1016/j.cub.2009.01.065

5. Blau, V., Reithler, J., van Atteveldt, N., Seitz, J., Gerretsen, P., Goebel, R. \& Blomert, L. (2010). Deviant processing of letters and speech sounds as proximate cause of reading failure: a functional magnetic resonance imaging study of dyslexic children. Brain, 133, 868-879. http://dx.doi. org/10.1093/brain/awp308

6. Bogliotti, C. (2005). Perception allophonique et dyslexie. Thèse de Doctorat en Linguistique, Université Denis Diderot - Paris 7.

7. Bogliotti, C. \& Serniclaes, W. (2011). Développement d'un instrument multimédia de remédiation de la dyslexie. JCP4, Strasbourg.

8. Bogliotti, C., Serniclaes, W., Messaoud-Galusi, S. \& Sprenger-Charolles, L. (2008). Discrimination of speech sounds by dyslexic children: Comparisons with chronological age and reading level controls. Journal of Experimental Child Psychology, 101, 137-175. http://dx.doi. org/10.1016/j.jecp.2008.03.006

9. Bouton, S., Serniclaes, W., Colé, P. \& Bertoncini, J. (accepted). Categorical perception of speech sounds in French-speaking children with cochlear implant. Journal of Speech, Language, and Hearing Research.

10. Brandt, J. \& Rosen, J.J. (1980). Auditory Phonemic Perception in Dyslexia : Categorical Identification and discrimination of stop consonants. Brain and Language, 9, 324-337. http://dx.doi.org/10.1016/0093-934X(80)90152-2

11. Burnham, D. (2003). Language specific speech perception and the onset of reading. Reading and Writing: An Interdisciplinary Journal, 16, 573-609. http://dx.doi. org/10.1023/A:1025593911070

12. Chobert, J. (2011). L'influence de l'apprentissage de la musique sur le traitement pré-attentif des syllabes chez les enfants normo-lecteurs et dyslexiques. Thèse de doctorat en Neurosciences. Université de la Méditerranée.

13. Clements, G.N. (1985). The geometry of phonological features. Phonology, 2, 225-252. http://dx.doi.org/10.1017/ S0952675700000440

14. Collet, G., Serniclaes, W., Colin, C. \& Leybaert, J. (2011). Improvement of the French voicing categorical perception in children with SLI after phonological auditory training. Escop.

15. Dehaene-Lambertz, G., Pallier, C., Serniclaes, W., SprengerCharolles, L., Jobert, A. \& Dehaene, S. (2005). Neural correlates of switching from auditory to speech perception. NeuroImage, 24, 21-33. http://dx.doi.org/10.1016/j.neuroimage.2004.09.039

16. Démonet, J.-F., Taylor, M.J. \& Chaix, Y. (2004). Developmental dyslexia. The Lancet, 363, 1451-1460. http://dx.doi. org/10.1016/S0140-6736(04)16106-0

17. Dufor, O., Serniclaes, W., Sprenger-Charolles, L. \& Démonet, J.-F. (2007). Top-down processes during auditory phoneme categorization in dyslexia: A PET study. NeuroImage, 34, 1692-1707. http://dx.doi.org/10.1016/j.neuroimage.2006.10.034

18. Dufor, O., Serniclaes, W., Sprenger-Charolles, L. \& Démonet, J.-F. (2009). Left pre-motor cortex and allophonic speech perception in dyslexia: A PET study. NeuroImage, 46, 241-248. http://dx.doi.org/10.1016/j.neuroimage.2009.01.035 
19. Eilers, R. E., Gavin, W. \& Wilson, W. (1979). Linguistic experience and phonetic perception in infancy: a cross-linguistic study. Child Development, 50, 14-18. http://dx.doi. org/10.2307/1129035

20. Ganong, W.F. III \& Keating, P. (1981). Selective adaptation of VOT in Polish. Journal of the Acoustical Society of America, 69, S115. http://dx.doi.org/10.1121/1.386483

21. Guenther, F.H. \& Bohland, J.W. (2002). Learning sound categories: A neural model and supporting experiments. Acoustical Science \& Technology, 23, 213-220. http:// dx.doi.org/10.1250/ast.23.213

22. Guenther, F.H., Husain, F.T., Cohen, M. A. \& Shinn-Cunningham, B. G. (1999). Effects of categorization and discrimination training on auditory perceptual space. Journal of the Acoustical Society of America, 106, 2905- 2912. http://dx.doi.org/10.1121/1.428112

23. Harnad, S. (1987). Categorical Perception. Cambridge: University Press.

24. Harm, M. W. \& Seidenberg, M. S. (1999). Phonology, reading acquisition, and dyslexia: Insights from connectionist models. Psychological Review, 106, 491-528. http://dx.doi. org/10.1037/0033-295X.106.3.491

25. Hickok, G. \& Poeppel, D. (2007). The cortical organization of speech processing. Nature Reviews Neuroscience, 8 , 393-402. http://dx.doi.org/10.1038/nrn2113

26. Hoonhorst, I., Colin, C., Markessis, E., Radeau, M., Deltenre, P. \& Serniclaes, W. (2009b). French native speakers in the making: from language-general to language-specific voicing boundaries. Journal of Experimental Child Psychology, 104, 353-366. http://dx.doi.org/10.1016/j. jecp.2009.07.005

27. Hoonhorst, I., Serniclaes, W., Collet, G., Colin, C., Markessis, E., Radeau, M. \& Deltenre, P. (2009a). The acoustic correlates of voicing perception in French. Clinical Neurophysiology, 120, 897-903. http://dx.doi.org/10.1016/j. clinph.2009.02.174

28. Hoonhorst, I., Medina, V., Colin, C., Markessis, E., Radeau, M., Deltenre P. \& Serniclaes, W. (2011). The development of categorical perception: comparisons between voicing, colors and facial expressions. Speech Communication, 53, 417-430. http://dx.doi.org/10.1016/j.specom.2010.11.005

29. Hurford, D.P. (1990).Training Phonemic Segmentation Ability with a Phonemic Discrimination Intervention in Second- and Third-Grade Children with Reading Disabilities. Journal of Learning Disabilities, 23, 564-569. http:// dx.doi.org/10.1177/002221949002300906

30. Jakobson, R. (1973). Essais de Linguistique Générale. Paris: Editions de Minuit.

31. Jamieson, D.G. \& Morosan, D.E. (1986). Training nonnative speech contrasts in adults: Acquisition of the Eng-

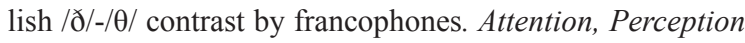
and Psychophysics, 40, 205-215. http://dx.doi.org/10.3758/ BF03211500
32. Lasky, R. E., Syrdal-Lasky, A. \& Klein, R. E. (1975). VOT discrimination by four to six and a half months old infants from Spanish environments. Journal of Experimental Child Psychology, 20, 215-225. http://dx.doi.org/10.1016/00220965(75)90099-5

33. Liberman, A. M., Harris, K. S. Hoffman, H. S. \& Griffith, B. C. (1957). The discrimination of speech sounds within and across phoneme boundaries. Journal of Experimental Psychology, 54, 358-368. http://dx.doi.org/10.1037/ $\underline{\mathrm{h} 0044417}$

34. Lisker, L. \& Abramson,A.S. (1970). The voicing dimension: some experiments in comparative phonetics. Proceedings of the 6th International Congress of Phonetic Sciences, Prague 1967; Prague: Academia; 563-567.

35. Luque, J.L., Serniclaes, W., López-Zamora, M., Bordoy, S., Giménez, A., Rosales, V. y Varona, S. (2011). Exploring Categorical Perception Deficit in Spanish Dyslexics Children. 10th International Symposium of Psycholinguistics. Donostia, Spain.

36. Majerus, S., Poncelet, M., Bérault, A., Audrey, S., Zesiger, P., Serniclaes, W. \& Barisnikov, K. (2011). Evidence for atypical categorical speech perception in Williams syndrome. Journal of Neurolinguistics 24, 249-267. http:// dx.doi.org/10.1016/j.jneuroling

37. McMurray, B., Tanenhaus, M. K. \& Aslin, R. N. (2002). Gradient effects of within-category phonetic variation on lexical access. Cognition, 86, B33-B42. http://dx.doi. org/10.1016/S0010-0277(02)00157-9

38. Medina, V., Hoonhorst, I., Bogliotti, C. \& Serniclaes, W. (2010). Development of voicing perception in French: Comparing adults, adolescents and children. Journal of Phonetics, 38, 493-503. http://dx.doi.org/10.1016/j. wocn.2010.06.002

39. Medina, V. \& Serniclaes, W. (2009). Consecuencias de la categorización fonológica sobre la lectura silenciosa de niños sordos con implante coclear. Revista de Logopedia, Foniatria y Audiologia, 29, 186-194. http://dx.doi. org/10.1016/S0214-4603(09)70027-0

40. Mitterer, H. (2006). On the causes of compensation for coarticulation: evidence for phonological mediation. Perception and Psychophysics. 68, 1227-1240. http://dx.doi. org/10.3758/BF03193723

41. Myers, E. B., Blumstein, S. E., Walsh, E. \& Eliassen, J. (2009). Inferior frontal regions underlie the perception of phonetic category invariance. Psychological Science, 20, 895-903. http://dx.doi.org/10.1111/j.14679280.2009.02380.x

42. Noordenbos, M., Segers, E., Mitterer, H., Serniclaes, W. \& Verhoeven, L. (2010). Allophonic perception in preschoolers. SSSR (Soc. for the Scientific Study of Reading) Conference Berlin.

43. Noordenbos, M., Segers, E., Mitterer, H., Serniclaes, W. \& Verhoeven, L. (submitted). Neural evidence of allophonic 
perception in children at risk for dyslexia.

44. Pisoni, D.B. (1977). Identification and discrimination of the relative onset time of two components tones: implications for voicing perception in stops. Journal of the Acoustical Society of America, 61, 1352-1361. http://dx.doi. org/10.1121/1.381409

45. Ramus, F. \& Szenkovits, G. (2008). What phonological deficit? The Quarterly Journal of Experimental Psychology, 61, 129-141. http://dx.doi.org/10.1080/17470210701508822

46. Ruff, S., Cardebat, D., Marie, N. \& Démonet, J.-F. (2002). Enhanced response of the left frontal cortex to slowed down speech in dyslexia: An fMRI study. Neuroreport, 13, 12851289. http://dx.doi.org/10.1097/00001756-20020719000014

47. Remez, R.E., Rubin, Ph., Pisoni, D.B. \& Carrell, T.D. (1981) Speech perception without traditional speech cues. Science, 212, 947-950. http://dx.doi.org/10.1126/science. 7233191

48. Ruff, S., Marie, N., Celsis, P., Cardebat, D. \& Démonet, J.F. (2003). Neural substrates of impaired categorical perception of phonemes in adult dyslexics: An fMRI study. Brain and Cognition, 53, 331-334. http://dx.doi.org/10.1016/ S0278-2626(03)00137-4

49. Serniclaes, W. (1987). Etude expérimentale de la perception du trait de voisement des occlusives du français [Experimental study of the perception of the voicing feature in French stop consonants]. Unpublished Doctoral Dissertation. Université Libre de Bruxelles.

50. Serniclaes, W. (2011). Features are phonological transforms of natural boundaries. In G.N. Clements and R. Ridouane (Eds.) Cognitive, physical and developmental bases of distinctive speech categories (pp. 237-260). Philadelphia: John Benjamins.

51. Serniclaes, W., \& Geng, C. (2009). Cross-linguistic trends in the perception of place of articulation in stop consonants: A comparison between Hungarian and French. In F. Pellegrino, E. Marsico, I. Chitoran \& C. Coupé, C. (Eds). Approaches to phonological complexity (pp. 241-266). The Haghe: Mouton de Gruyter, Phonology \& Phonetics Series.

52. Serniclaes, W., Sprenger-Charolles, L., Carré, R. \& Démonet, J.F. (2001). Perceptual discrimination of speech sounds in dyslexics. Journal of Speech Language and Hearing Research, 44, 384-399. http://dx.doi.org/10.1044/10924388(2001/032)

53. Serniclaes, W., Van Heghe, S., Mousty, Ph., Carré, R. \& Sprenger-Charolles, L. (2004). Allophonic mode of speech perception in dyslexia. Journal of Experimental Child Psychology, 87, 336-361. http://dx.doi.org/10.1016/j. jecp.2004.02.001

54. Serniclaes, W., Ventura, P, Morais, J. \& Kolinsky, R. (2005). Categorical perception of speech sounds in illiterate adults. Cognition, 98, B35-B44. http://dx.doi.org/10.1016/j.cognition.2005.03.002
55. Share D.L. (1995). Phonological recoding and self-teaching: Sine qua non of reading acquisition. Cognition, 55, 151-218. http://dx.doi.org/10.1016/0010-0277(94)00645-2

56. Sinnott, J.S. \& Gilmore, C.S. (2004). Perception of placeof-articulation information in natural speech by monkeys versus humans. Perception \& Psychophysics, 66, 13411350. http://dx.doi.org/10.3758/BF03195002

57. Sprenger-Charolles, L., Colé, P. \& Serniclaes, W. (2006). Reading Acquisition and Developmental Dyslexia. New York: Psychology Press (Developmental Essays).

58. Treisman, (1999). There are two types of psychometric function: A theory of cue combination in the processing of complex stimuli with implications for categorical perception. Journal of Experimental Psychology (General), 128, 517-546.

59. Van Beinum, F.J., Schwippert, C.E., Been, P.H., van Leeuwen, T.H. \& Kuijpers, C.T.L. (2005). Development and application of a $/ \mathrm{bAk} /-\mathrm{dAk} /$ continuum for testing auditory perception within the Dutch longitudinal dyslexia study. Speech Communication, 47, 124-142. http://dx.doi. org/10.1016/j.specom.2005.04.003

60. Vandermosten, M., Boets, B., Luts, H., Poelmans, H., Golestani, N., Wouters, J. \& Ghesquière, P. (2010). Adults with dyslexia are impaired in categorizing speech and nonspeech sounds on the basis of temporal cues. Proceedings of the National Academy of Sciences of the United States of America, 107, 10389-10394. http://dx.doi.org/10.1073/ pnas.0912858107

61. Vandermosten, M., Boets, B., Luts, H., Poelmans, Wouters, J. \& Ghesquière, P. (2011). Impairments in speech and nonspeech sound categorization in children with dyslexia are driven by temporal processing difficulties. Research in Developmental Disabilities, 32, 593-603. http://dx.doi. org/10.1016/j.ridd.2010.12.015

62. Van Heghe, S. (2001). Evaluation comparative des effets de deux entraînements à la discrimination catégorielle sur les habilités de perception catégorielle et métaphonologiques des enfants dyslexiques. Mémoire de Licence en Sciences Psychologiques et de 1'Education, ULB.

63. Veuillet, E., Magnan, A., Ecalle, J., Thai-Van, H. \& Collet, L. (2007). Auditory processing disorder in children with reading disabilities: effect of audiovisual training. Brain, 130, 2915-2928. http://dx.doi.org/10.1093/brain/awm235

64. Williams, L. (1977). The voicing contrast in Spanish. Journal of Phonetics, 5, 16984.

65. Zobouyan, C., Bertoncini, J. \& Serniclaes, W., (2010). Déficit de perception catégorielle chez les enfants dysphasiques. 28èmes Journées d'Etudes sur la Parole (JEP), Mons, Juin 2010.

Fecha de recepción: 15 de marzo de 2011

Fecha de recepción de la versión modificada: 7 de abril de 2011 Fecha de aceptación: 2 de mayo de 2011 